\title{
CHAMGES IN BAR VELOCITY AND MUSCULAR ACTIVITY DURING THE BENCH PRESS IN RELATION TO THE LOAD LIFTED
}

\author{
Artur Gołaś, 1, A, B, E Adam Maszczyk, 2, A, C, D, E Henryk Król, ,, A, B Michał Wilk, , C, D \\ Petr Statsny, ${ }^{3, D}$, E Miroslav Petr, ${ }^{4, D}$ Grzegorz Wróbel ${ }^{5, A, C, D}$
}

\footnotetext{
${ }^{1}$ Department of Sports Training, The Jerzy Kukuczka Academy of Physical Education, Katowice, Poland

2 Department of Statistics and Methodology, The Jerzy Kukuczka Academy of Physical Education, Katowice, Poland

${ }^{3}$ Palacky University in Olomouc, Faculty of Physical Culture, Olomouc, Czech Republic

${ }^{4}$ Department of Physiology and Biochemistry, Faculty of Physical Education and Sport, Charles University in Prague, Czech Republic

${ }^{5} \mathrm{PhD}$ student. The Jerzy Kukuczka Academy of Physical Education, Katowice, Poland

${ }^{6}$ Department of Motor Behavior, The Jerzy Kukuczka Academy of Physical Education, Katowice, Poland

${ }^{\text {A }}$ Study Design; ${ }^{B}$ Data Collection; ' ${ }^{\text {S }}$ tatistical Analysis; ${ }^{D}$ Manuscript Preparation; EFunds Collection
}

\author{
Address for correspondence: \\ Adam Maszczyk Ph.D. Associated Professor \\ The Jerzy Kukuczka Academy of Physical Education \\ Department of Statistics and Methodology \\ Mikołowska 72a, 40-065 Katowice, Poland \\ E-mail: a.maszczyk@awf.katowice.pl
}

\begin{abstract}
Ahstract. The purpose of the study was to determine velocity changes in relation to the load lifted along with their muscular activity. Twenty athletes representing different sport disciplines, familiar with the flat bench press took part in the research project. The ANOVA analysis revealed a significantly higher effect on range of mean velocity $(F=128.34 ; \eta 2=1.22$ with $p=0.001)$ and maximal velocity $(F=73.31 ; \eta 2=0.81$ with $p=0.001)$ to measured loads $(70 \div 100 \% 1-R M)$ in ascending phase. Tukey's post-hoc tests revealed a statistically significant difference between $70 \%$ and $100 \% 1$-RM loads, in mean velocity with $p=0.001$ and maximal velocity with $p=0.001$. Similarly the results revealed a statistically significant difference between $80 \%$ and $100 \% 1$-RM loads in mean velocity $(p=0,012)$ and maximal velocity $(p=0,021)$ during the ascending phase. Relationship between velocity and the muscles activity indicates that the anterior deltoid and latissimus dorsi muscles show synergy and a significant relationship for loads 90 and 100\% 1-RM. The triceps brachii muscle shows a higher relationship with velocity of the barbell for loads $70 \%$ and $80 \%$, then the relationship of the pectoralis major muscle with velocity only for $90 \% 1-\mathrm{RM}$.
\end{abstract}

Key WOrll:: sticking region, EMG, bar velocity

\section{Introduction}

The bench press is one of the most popular exercises used in strength training for the upper body. A successful bench press lift is performed when the barbell is first lowered to the chest and then moved to a fully extended position. The bench press consists of two phases: the ascending and descending phase. The ascending phase seems more significant for bench press performance (Barnett et al. 1995; Requena et al. 2005; Welsch et al. 2005; 
Van den Tillaar and Ettema 2009; Król et al. 2010). Several studies have investigated the kinematics of the bench press, and have shown that there is a sticking region (SR) during maximal lifts (Madsen and McLaughlin 1984). In this region, the pushing force is less than gravity on the barbell, leading to a deceleration of the barbell. It is defined as the movement region from peak velocity $\left(V_{\text {max }}\right)$ to the first local minimum velocity $\left(V_{\text {min }}\right)$ of the upward barbell movement (van den Tillaar and Ettema 2009). Van den Tillaar and Ettema (2009) found that the muscle activity of only the agonistic pectoralis major muscles and the anterior part of the deltoid muscles are responsible for the SR. They proposed that the start of a SR occurs, not because of a lack of strength, but due to diminishing of enhanced force (i.e., potentiation induced by the immediately preceding eccentric contraction) at the start of the concentric movement. When this strength capacity diminishes, a delayed neural reaction occurs (Walshe et al. 1998; Santana et al. 2007), enhancing the muscle activity level, so that the resultant force matches the demands of the attempt. Thus, the delay in neural activity would be the cause of the SR, whereas the increase itself results in the overcoming of the sticking region (Van den Tillaar and Ettema 2009).

In the literature there is a lack of information concerning the changes of velocity of the barbell in relation to the activity of the muscles at different loads. There is significantly more data regarding only the velocity of the bar during the bench press. Duffey and Challis (2007) studied the effects of fatigue on this kinematic parameter. On the other hand Pearson et al. (2009) assessed the velocity changes resulting from increased load of the bar on a Smith machine. Similar studies were conducted by Sakamoto and Sinclair (2006), yet the barbell was pressed slowly, moderately, very fast and explosively. This information is of great significance in assessment of the technique of the bench press motion. However, of more importance is the knowledge related to the dynamics of the movement, what is associated with the activity of the muscles engaged in this motor task.

The purpose of the study was to determine velocity changes in relation to the load lifted along with their muscular activity.

\section{Methods}

\section{Participants}

Twenty athletes representing different sport disciplines, familiar with the flat bench press took part in the research project. Their average age, body mass, height and 1-RM equalled respectively: (age: $24.7 \pm 0.9 \mathrm{yrs}$; body mass: $80.2 \pm 8.6 \mathrm{~kg}$; height: $176.8 \pm 8.0 \mathrm{~cm}$; 1-RM (one repetition maximum): $107.1 \pm 19.4 \mathrm{~kg}$ ). The participants were informed about the nature of this study and, prior to data collection, they were required to sign a declaration form for participation in accordance with the Helsinki Declaration. The participants did not perform any resistance training of the upper body 72 hours prior to testing. The research project was approved by the Bioethics Committee of the Academy of Physical Education in Katowice.

\section{Exercise protocol}

The exercise protocol included a warm-up and the main session. The participants followed the same warm up procedure as used by Saeterbakken et al. (2011). They started with a general warm-up of 15 min, followed by a specific warm-up, which included four sets of the bench press: 1) twenty repetitions at $30 \%$ of 1-RM, 2) twelve repetitions at $50 \%$ of $1-\mathrm{RM}, 3$ ) six repetitions at $70 \%$ of $1-\mathrm{RM}$ and 4 ) one repetition at $85 \%$ of $1-\mathrm{RM}$. The percentage of the 1-RM was estimated based on self-reported 1-RM of the participants. The self-reported 1RM was set 
according to the information given by the participants on maximal lifts performed in the previous three weeks (Reynolds et al. 2006).

The main session included four sets of one repetition of the flat bench press, using $70,80,90$, and $100 \%$ of the estimated maximum load. After approaching the estimated maximum load, the resistance was increased, until the subjects could not lift the bar. For velocity and electromyography analysis bench press attempts with the following loads were considered: 70, 80, 90, 100\% 1-RM. In the main session each of the subjects performed from 6 to 9 sets to reach his individual 1 RM. Five minute rest periods were provided between sets. After cessation of all the bench press attempts, three $3 \mathrm{~s}$ trials of static effort in the bench press position were performed to normalize MVIC.

The exercise protocol was conducted with free weights, with an Olympic bar and a flat bench. During the execution of the bench press, the back and head of the participants remained on the bench, their knees flexed at approximately 90 degrees, while the feet were placed flat on the ground. The width of the bar grip equaled $81 \mathrm{~cm}$ between the index fingers, what is the greatest value permitted by the International Powerlifting Federation. Two spotters were used during all bench press attempts to provide safety for the participants. The subjects lowered the bar in a controlled manner, until touching the chest, and then performed the pressing action, without stopping until arms were fully extended.

\section{0 kinematics and electromyography}

In following research a complex analysis of the movement was performed, with the use of the BTS SMART-E system (BTS Bioengineering, Italy) which consisted of six infrared cameras $(120 \mathrm{~Hz})$ and a wireless module to measure bioelectric activity of the major muscles involved in the bench press.

The EMG signals were measured by a Pocket EMG System (BTS Company, Italy). All active channels were the same, and the measuring range was fitted to the subject (typically $\pm 10 \mathrm{mV}$ ). EMG activity of 4 muscles was measured: pectoralis major, anterior deltoid, triceps brachii (long head) and latisimuss dorsi. Before placing the gel coated self-adhesive electrodes (Dri-Stick Silver circular sEMG Electrodes AE-131, NeuroDyne Medical, USA), the skin was shaved, abraded and washed with alcohol. The electrodes (11 mm contact diameter and a $2 \mathrm{~cm}$ center-to-center distance) were placed along the presumed direction of the underlying muscle fiber according to the recommendations by SENIAM (Hermens et al. 2000; Lehman et al. 2006). To minimize noise induced from external sources, the EMG signal was amplified and filtered using a preamplifier located as near the pickup point as possible. The EMG signals were sampled at a rate of $1000 \mathrm{~Hz}$. Signals were filtered with a cut off frequency of $8 \mathrm{~Hz}$ and $450 \mathrm{~Hz}$.

\section{Statistical analysis}

After calculating the average values $(x)$ and standard deviations $( \pm S D)$, particular groups of presses with different loads were compared by ANOVA. The statistical analysis was directed at determining dependent variables differentiated by the independent variable (Maszczyk et al. 2011, 2012). Effect sizes (partial eta squared $\eta 2$ and Cohen's d) were reported for results, where appropriate. Parametric effect sizes were defined as large $d>0.8$, moderate as between 0.8 and 0.5 , and a small defined as $<0.5$ (Cohen 1988). Between all of the variables, correlation coefficients were determined with Pearson's rank order test. Statistical significance was set at $p<0.05$. All statistical analyses were performed using Statistica 9.1 with neural network module, and Microsoft Office - Excel 2010 packets. 


\section{Results}

Changes in bench press velocity according to different loads are presented in Figure 1.
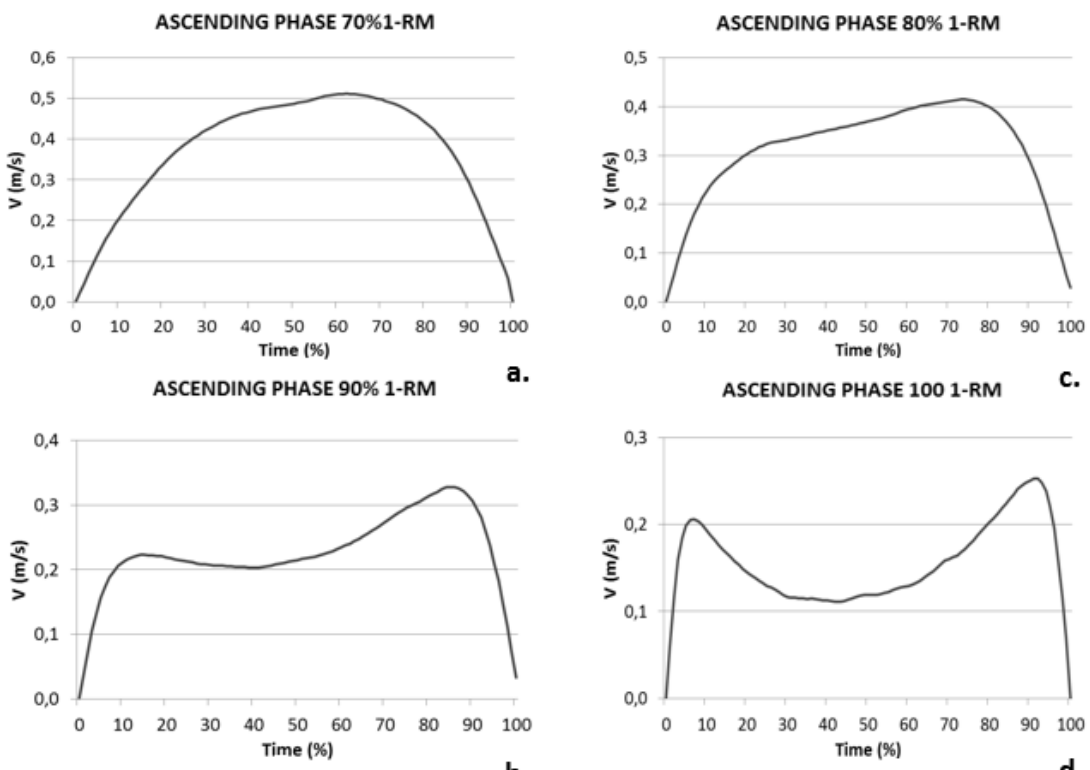

b.

d.

Figure 1. Velocity of the bar during the ascending phase in the flat bench press with a load of a. 70\% 1-RM; b. 80 1-RM; c. 90 1-RM; d. $100 \%$ 1-RM

Multivariate analysis of variance (ANOVA) revealed a significantly higher effect on range of mean velocity $(F=128.34 ; \eta 2=1.22$ with $p=0.001)$ and maximal velocity $(F=73.31 ; \eta 2=0.81$ with $p=0.001)$ to measured loads $(70 \div 100 \% 1-\mathrm{RM})$ in ascending phase. Tukey's post-hoc tests revealed a statistically significant difference between $70 \%$ and $100 \% 1$-RM loads, in mean velocity with $p=0.001$ and maximal velocity with $p=0.001$. Similarly the results revealed a statistically significant difference between $80 \%$ and $100 \% 1-$ RM loads in mean velocity $(p=0.012)$ and maximal velocity $(p=0.021)$ during the ascending phase.

Table 1. Bioelectrical activity muscles (MVIC): pectoralis major, deltoid, triceps brachii and latissimus dorsi during ascending phase flat bench pressing on the load of 70, 80, 90 and 100\% 1-RM

\begin{tabular}{ccccc}
\hline Value of the load & Pectoralis major & Deltoid & Triceps brachii & Latisimuss Dorsi \\
\hline $70 \% 1$-RM & $97.6 \pm 26,6$ & $78.8 \pm 23.6$ & $64.8 \pm 28$ & $59.6 \pm 17.3$ \\
$80 \%$ 1-RM & $98.2 \pm 26.4$ & $83.8 \pm 22.2$ & $78.8 \pm 32.7$ & $69.6 \pm 20.7$ \\
$90 \% 1-\mathrm{RM}$ & $101.4 \pm 21.1$ & $94.6 \pm 23.2$ & $99.1 \pm 37$ & $83.9 \pm 26.6$ \\
$100 \% 1-\mathrm{RM}$ & $98.7 \pm 19.2$ & $106.2 \pm 20.9$ & $118.9 \pm 37.7$ & $105.5 \pm 28.9$ \\
$\bar{X} \pm$ SD & $99.0 \pm 1.7$ & $90.8 \pm 12.2$ & $90.4 \pm 23.7$ & $79.7 \pm 19.9$ \\
\hline
\end{tabular}


Values of bioelectrical activity of the evaluated muscles (MVIC): pectoralis major, deltoid, triceps brachii and latissimus dorsi during the ascending phase of the flat bench press with the load of 70, 80, 90 and 100\% 1-RM are presented in Table 1.

The most statistically significant positive correlations between MVIC and the pectoralis major. deltoid, triceps brachii and Latisimuss dorsi muscles and velocity during the ascending phase of the bench press are presented in Table 2. Considering the relationship between the velocity of the bar and the bioelectrical activity of the main muscle groups participating in the bench press, in attempts with a load of 70 and $80 \%$ of $1 \mathrm{RM}$, the correlation coefficients were very high and positive only in case of the triceps brachii. Also a significant, positive correlation with bar velocity was observed for the pectoralis major muscle with the load of $90 \%$ of $1 \mathrm{RM}$.

\section{Discussion}

In bench press attempts with a load of 70 and $80 \% 1 \mathrm{RM}$ only one peak of vertical velocity was registered during the ascending phase of the movement. On the contrary during lifts with 90 and 100\% 1-RM, a sticking point appeared, during which the velocity reached minimum values (Duffey and Challis 2007; Van den Tillaar and Ettema 2009; Saeterbakken et al. 2011). Van Den Tillaar and Ettema (2009) searching for the causes of decreased velocity during the bench press movement, conclude that the sticking point is not caused by the lack of strength itself, but a delay of neural signals from the triceps brachii to the pectoralis major and deltoid muscles. The vertical component of bar velocity decreases with a rise in external load, in relations to mean values in the ascending phase as well as to maximum velocity (peak velocity).

The most significant differences for both mean and maximal bar velocity occur between 70 and 100\% 1-RM, as well as between 80 and 100\% 1RM. These differences are associated with a decrease in velocity and the appearance of the sticking region. Deceleration was higher in lifts with the maximal load, which may have been caused by diminishing potentiation of the contractile elements (Walshe et al. 1998; Konrad 2005; Reynolds et al. 2006; Trebs et al. 2010). Furthermore, the difference in lifted weight was strongly associated with a significant increase in bioelectrical activity of all the studied muscles.

The relationship between the velocity of the bar and the bioelectrical activity of the studied muscles, indicate that for loads 70 and $80 \%$ 1-RM, an increase in muscular activity of the triceps brachii causes an increase of bar velocity during the bench press.

The increase in bar velocity for the $90 \%$ of $1 \mathrm{RM}$ load is caused by increased activity of the pectoralis major, deltoid and latisimuss dorsi muscles.

These relationships change significantly during lifts with a load of $100 \% 1 \mathrm{RM}$, where the decrease in bar velocity is caused by an increase in the bioelectrical activity of the deltoid and latisimuss dorsi muscles. This may indicate that a higher activity of these muscle groups is significantly related to flat bench press performance.

In the conducted research the average bar velocity decreased respectively from $0.515 \pm 0.09 \mathrm{~m} / \mathrm{s}$ with a load of $70 \% 1$-RM through $0.415 \pm 0.09 \mathrm{~m} / \mathrm{s}$ with $80 \% 1$-RM to $0.325 \pm 0.06 \mathrm{~m} / \mathrm{s}$ with $90 \% 1-R M$ down to $0.251 \pm 0.07 \mathrm{~m} / \mathrm{s}$ with the maximum load.

It must be indicated that during the lift with 90 and 100\% 1RM the peak bar velocities are reached for the second time during the ascending phase of the bench press motion. During the minimum bar velocity in the lift with $100 \%$ load, the bar traveled $37 \%$ of the entire path of the movement. 
In case of Landers et al. research (1985) it was 41.8\%, in Madsens and McLaughlins studies (1984) it reached $50.2 \%$, while Elliott et al. (1989) recorded a value of $47.9 \%$. Duffey and Challis (2007) evaluated the effects of fatigue on bar kinematics during the bench press. In 18 subjects the peak bar velocity during the first repletion of the bench press with a load of $75 \%$ 1-RM equaled $0.46 \pm 0.11 \mathrm{~m} / \mathrm{s}$ while in the last repletion of this set it dropped to $0.25 \pm 0.08 \mathrm{~m} / \mathrm{s}$. The tested subjects in this experiment performed on the average $10 \pm 3$ repetitions. In the attempt with $100 \%$ 1-RM load peak bar velocity reached $0.35 \mathrm{~m} / \mathrm{s}$, and was attained at the end of the ascending phase.

\section{Practical Implications}

Relationship between velocity and the muscles activity indicates that the anterior deltoid and latissimus dorsi muscles show synergy and a significant relationship for loads 90 and 100\% 1-RM. The triceps brachii muscle shows a higher relationship with velocity of the barbell for loads $70 \%$ and $80 \%$, then the relationship of the pectoralis major muscle with velocity only for $90 \% 1$-RM. Future studies in bench press should be conducted for loads over $100 \%$ 1-RM for determining the velocity changes and their relationship with muscles activity. During maximal loading (100\% 1-RM) the pectoralis major muscle decreases its activity in the ascending phase compared to lower loads. Most strength training references indicate that the pectoralis major is the most significant muscle, engaged in the flat bench press. The results of our research indicate that to lift heavy loads in the flat bench press the athletes must significantly develop the anterior deltoid and triceps brachii muscles. The bioelectrical activity of particular muscles changes significantly during different external load. The results and practical implications of this research conducted on the bench press may be applied to other resistance exercises.

\section{Acknowledgement}

The author's research is funded by a grants of Ministry of Science and Higher Education of Poland (NRSA2 02552 and NRSA3 03953).

\section{References}

Barnett C., Kippers V., Turner P. Effects of Variations of the Bench Press Exercise on the EMG Activity of Five Shoulder Muscles. Journal of Strength and Conditioning Research. 1995; 9 (4): 222-227.

Cohen J. Statistical power analysis for the behavioral sciences (2nd ed.). 1988; Hillsdale, NJ; Erlbaum.

Duffey M.J., Challis J.H. Fatique effects on bar kinematics during the bench press exercise. J. Hum. Kinet. 2007; 21: 33-40.

Elliott B.C., Wilson G.J., Kerr G.K. A biomechanical analysis of the sticking region in the bench press. Med. Sci. Sports Exerc. 1989; 21: 450-462.

Hermens H.J., Freriks B., Disselhorst-Klug C., Rau G. Development of recommendations for SEMG sensors and sensor placement procedures. J Elect Kin. 2000; 10 (5): 361-374

Konrad P. The ABC of EMG: A practical introduction to kinesiological electromyography. Version 1.0. Noraxon INC., USA, 2005; $29-33$.

Król H., Golas A., Sobota G. Complex analysis of movement in evaluation of flat bench press performance. Acta of Bioengineering and Biomechanics. 2010; 12 (2): 93-97.

Lander J.E., Bates B.T., Swahill J.A., Hamill J. A comparison between free-weight and isokinetic bench pressing. Med Sci Sports Exerc. 1985; 17 (3): 344-353.

Lehman G.J., MaCmillan B., Macintyre I., Chivers M., Fluter M. Shoulder muscle EMG activity during push up variations on and off a Swiss ball. Dynamic Medicine. 2006; 5: 7 .

Madsen N., McLaughlin T. Kinematic factors influencing performance and injury risk in the bench press exercise. Med Sci Sports Exerc. $1984 ; 16$ (4): 376-381. 
Maszczyk A., Roczniok R., Czuba M., Zając A., Waśkiewicz Z., Mikolajec K., Stanula A. Application of regression and neural models to predict competitive swimming performance. Perceptual \& Motor Skills. 2012; 114 (2): 610-624.

Maszczyk A., Zając A., Ryguła I. A Neural Network model approach to athlete selection. Sport Engineering. 2011; 13 : 83-93.

Pearson S.N., Cronin J.B., Hume P.A., Slyfield D. Kinematics and kinetics of the bench - press and bench - pull exercises in a strength - trained sporting population. Sport Biom. 2009; 8: 245-254.

Requena B., Zabala M., Ribas J., Ereline J., Paasuke M., Gonzalez-Badillo J.J. Effect of post-tetanic potentiation of pectoralis and triceps muscles on bench press performance. Journal of Strength and Conditioning Research. 2005; 19 (3): $622-627$.

Reynolds J.M., Gordon T.J., Robergs R.A. Predictions of one repetition maximum strength from multiple repetition maximum testing and anthropometry. Journal of Strength and Conditioning Research. 2006; 20 (3): 584-592.

Saeterbakken A., van den Tillaar R., Fimland M. A comparison of muscle activity and 1-RM strength of three chest-press exercises with different stability requirements. J Sports Sci. 2011; 29 (1): 1-6.

Sakamoto A., Sinclair P.J. Effect of movement Velocity on the relationship between training load and the numer of repetitions of bench press. J. Strength Cond. Res. 2006; 20 (3): 523-527.

Santana J.C., Vera-Garcia F.J., Mcgill S.M. A kinetic and electromyographic comparison of the standing cable press and bench press. Journal of Strength and Conditioning Research. 2007; 21 (4): 1271-1279.

Trebs A., Brandenburg J., Pitney W.A. An Electromyography Analysis of 3 Muscles Surrounding the Shoulder Joint During the Performance of a Chest Press Exercise at Several Angles. Journal of Strength \& Conditioning Research. 2010; 24 (7): 1925-1930.

Van Den Tillaar R., Ettema G. A comparison of successful and un successful attempts in maximal bench pressing. Medicine and Science in Sports and Exercise. 2009; 41 (11): 2056-2063.

Walshe A.D., Wilson G.J., Ettema G. Stretch shorten cycle as compared to isometric preload: contributions to enhanced muscular performance. J Appl Physiol. 1998; 84: 97-106.

Welsch E.A., Bird M., Mayhew J.L. Electromyographic activity of the pectoralis major and anterior deltoid muscles during three upperbody lifts. J. Strength Cond. Res. 2005; 19: 449-452.

Cite this apticle aS: Gołaś A., Maszczyk A., Król H., Wilk M., Statsny P., Petr M., Wróbel G. Changes in Bar Velocity and Muscular Activity During the Bench Press in Relation to the Load Lifted. Central European Journal of Sport Sciences and Medicine. $2015 ; 11$ (3): 95-101. 
